\title{
Review
}

\section{Physical activity during leisure time and primary prevention of coronary heart disease: an updated meta-analysis of cohort studies}

\author{
Francesco Sofia ${ }^{a, b}$, Andrea Capalbo ${ }^{a, b, c}$, Francesca Cesari ${ }^{a, b}$, \\ Rosanna Abbate ${ }^{a, b}$ and Gian Franco Gensini ${ }^{a, b, d}$
}

\begin{abstract}
aDepartment of Medical and Surgical Critical Care, Thrombosis Centre, Azienda Ospedaliero-Universitaria Careggi, ${ }^{b}$ Center for the Study at Molecular and Clinical Level of Chronic, Degenerative and Neoplastic Diseases to Develop Novel Therapies, University of Florence, ${ }^{\mathrm{C}}$ Institute of Sports Medicine and ${ }^{\mathrm{d}}$ Don Carlo Gnocchi Foundation, IRCCS, Florence, Italy
\end{abstract}

Received 8 August 2007 Accepted 14 September 2007

Background A vast body of evidence during the last decades has shown the clear preventive role of physical activity in cardiovascular disease. The real magnitude of the association between physical activity during leisure time (LTPA) and primary prevention of coronary heart disease (CHD) has, however, not been completely defined.

Design Meta-analysis of prospective cohort studies.

Methods Studies were included if they reported relative risks and their corresponding $95 \%$ confidence intervals $(\mathrm{Cl})$, for categories of LTPA in relation to CHD. The LTPA categories of the selected studies were grouped into three levels of intensity: high, moderate and low. The high level of physical activity was determined, to obtain a level of intensity attainable by the general population.

Results Data were available for 26 studies, incorporating 513472 individuals (20666 CHD events), followed up for 4-25 years. Under a random-effects model, the overall analysis showed that individuals who reported performing a high level of LTPA had significant protection against CHD [relative risk $0.73(95 \% \mathrm{Cl} 0.66-0.80), P<0.00001$ ]. A similar significant protection against CHD, for individuals who practised a moderate level of LTPA, has been also demonstrated [relative risk $0.88(95 \% \mathrm{Cl} 0.83-0.93), P<0.0001]$.

Conclusions The current meta-analysis reports significant protection against the occurrence of CHD resulting from moderate-to-high levels of physical activity. These results strengthen the recommendations of guidelines that indicate the protective effect against cardiovascular disease of physical activity profiles that are attainable by ordinary people. Eur $J$ Cardiovasc Prev Rehabil 15:247-257 @ 2008 The European Society of Cardiology

European Journal of Cardiovascular Prevention and Rehabilitation 2008, 15:247-257

Keywords: cardiovascular disease, coronary heart disease, exercise, meta-analysis, physical activity

\section{Introduction}

Cardiovascular disease (CVD) is the first leading cause of mortality in industrialized countries. Recent statistics report that more than one third of the world population die of CVD [1]. In particular, death from coronary heart

Correspondence to Dr Francesco Sofi, MD, PhD, Department of Medical and Surgical Critical Care, Thrombosis Centre, University of Florence, Azienda Ospedaliero-Universitaria Careggi, Viale Morgagni 85, 50134 Florence, Italy Tel: + 39055 7949420; fax: + 390557949418 ;

e-mail: francescosofi@gmail.com disease (CHD) accounts for more than $50 \%$ of all the causes of death due to CVD [1].

Over the last few decades, many epidemiologic studies performed on several different and large populations have demonstrated a clear protective effect of physical activity on CVD [2-4]. Some reviews and meta-analyses on the association between physical activity and CVD have been carried out. In 1990, Berlin and Colditz [5] applied the techniques of meta-analysis to data extracted from the 
published literature by Powell et al. [6]. In this manner, they demonstrated an increased relative risk (RR) of death from CHD for sedentary individuals, which was nearly twice that for active individuals. In 1992, Eaton [7] systematically reviewed all the epidemiological studies in relation to physical activity and to the primary prevention of CHD, by showing a significant and independent relationship between physical inactivity and CHD. Finally, a meta-analysis that included only studies performed in women recently demonstrated a beneficial dose-response relationship of physical activity on various CVD outcomes, especially CHD and stroke [8].

Despite this, however, the real magnitude of association between leisure-time physical activity (LTPA) and CHD has not been completely defined, as previous metaanalyses did not specifically target the role of LTPA in the primary prevention of CHD. In fact, over the last few decades, the type of physical activity conducted by the general population has progressively shifted from LTPA to work-related physical activity. Some of these metaanalyses analysed studies that included overall physical activity (leisure-time-related and work-related), and not just LTPA, as the measure of association. In addition, they evaluated CVD, and not just CHD, as a clinical outcome; sometimes the meta-analysis included studies that were sex-specific. Most importantly, since their publication, several relevant and updated data from cohort studies have been published.

We have, therefore, decided to conduct an updated systematic review, with a meta-analysis of all the available prospective cohort studies that examined the effect of LTPA on the primary prevention of CHD among men and women, considering that only LTPA can really be influenced by the recommendations of guidelines.

\section{Methods}

We developed a systematic literature search, to identify studies evaluating the association between LTPA and primary prevention of CHD. We searched the electronic databases MEDLINE (from 1966), EMBASE (from 1980), Science Citation Index (from 1994) and the Cochrane Systematic Review database up to May 2007, using a combined text word and MeSH search strategy for the terms 'physical activity' and 'physical exercise' in combination with 'cardiovascular disease', 'coronary artery disease', 'coronary heart disease', 'ischaemic heart disease', 'myocardial infarction' and 'acute coronary syndromes'. Furthermore, we reviewed reference lists of original, review articles and meta-analyses, to search for more studies.

For inclusion, studies had to fulfil the following criteria: they had to have a prospective cohort design; provide as a primary or secondary outcome the association between
LTPA and CHD and report relative risks (RR) and their corresponding 95\% confidence intervals (CI) of CHD, in relation to different categories of LTPA.

Studies were excluded if cross-sectional or case-control design was used; outcome was CVD or stroke, and not CHD; physical activity was evaluated only as occupational activity, or as an overall index that included occupational physical activity and if statistical analysis was not adequate, that is, reporting of insufficient information to compute an estimate of risk or its standard error, for all the categories of physical activity. If multiple published reports from the same study cohort were available, we included only that with the most recent updated and detailed information for both outcomes and physical activity categories.

All data were independently extracted by two investigators (F.S. and A.C.) using a standardized data-extraction tool, and were entered into separate databases. Results were compared, and disagreements were resolved through discussions with a third investigator (F.C.). Relevant data included the first author's name, year of publication, cohort name, country of origin of the centres, number of participants and number of CHD events, duration of follow-up, age and sex of participants, main outcome, methods used to measure LTPA, RRs or hazard ratios of CHD and corresponding 95\% CIs for all categories of LTPA and covariates adjusted in the statistical analysis. The study quality of each article was checked.

As the quality varied widely among the studies reviewed, a summary score of study quality was developed; this score was adapted from Powell et al. [6], to evaluate three aspects of each study: assessment of LTPA, assessment of outcome and epidemiologic method. Each category was assigned $0-2$ points. A maximum of six points was given if all the three categories were fully satisfied.

\section{Statistical analysis}

Data were analysed using Review Manager (RevMan) software for Windows (version 4.2, http://www.cc-ims.net) RevMan) by the Cochrane Collaboration, 2003, and Statistical Package for Social Sciences (SPSS) software for Windows (version 13.0).

As the studies included in this meta-analysis reported physical activity using different methods and determined the categories of activity in different ways, we distinguished three levels of LTPA: high, moderate and low. The category with the highest level of LTPA was determined mainly by taking into consideration the second-highest category of LTPA from the studies: this was done to exclude the possible confounding effect of vigorous LTPA on disease and to obtain a level of intensity that could be attained by the general 


\begin{tabular}{|c|c|c|c|c|c|c|c|c|c|c|c|}
\hline Source, year (cohort) & $\begin{array}{l}\text { Country } \\
\text { (baseline } \\
\text { year) }\end{array}$ & $\begin{array}{l}\text { Number of } \\
\text { events/ } \\
\text { number of } \\
\text { participants }\end{array}$ & $\begin{array}{l}\text { Follow-up, } \\
\text { years }\end{array}$ & Age, years & Sex & Outcome & $\begin{array}{l}\text { Assessment of physical } \\
\text { activity }\end{array}$ & $\begin{array}{l}\text { Physical activity } \\
\text { categories }\end{array}$ & $\begin{array}{l}\text { Adjusted RR } \\
(95 \% \mathrm{Cl})\end{array}$ & Adjustment & Study quality \\
\hline \multirow[t]{2}{*}{$\begin{array}{l}\text { Rodriguez et al. (Honolulu } \\
\text { Heart Program) [14] }\end{array}$} & USA (1965) & 789/7074 & 23 & $45-68$ & M & $\mathrm{CHD}$ & $\begin{array}{l}\text { Index is based on the } \\
\text { average number of hours } \\
\text { spent per day in activ- } \\
\text { ities of various levels, } \\
\text { multiplied by weights } \\
\text { derived from the oxygen } \\
\text { consumption needed for } \\
\text { each level }\end{array}$ & Lowest tertile & 1.00 & $\begin{array}{l}\text { Age, body mass index, } \\
\text { smoking habit, hyper- } \\
\text { tension, total cholesterol, } \\
\text { diabetes and alcohol }\end{array}$ & $6(2 ; 2 ; 2)$ \\
\hline & & & & & & & & $\begin{array}{l}\text { Middle tertile } \\
\text { Highest tertile }\end{array}$ & $\begin{array}{l}1.07(0.90-1.23) \\
0.95(0.80-1.14)\end{array}$ & & \\
\hline $\begin{array}{l}\text { Lakka et al. (Kuopio } \\
\text { Ischemic Heart Disease } \\
\text { Risk Factor Study) [15] }\end{array}$ & $\begin{array}{l}\text { Finland } \\
(1984)\end{array}$ & $42 / 1166$ & 4.9 & $42-61.2$ & M & MI & $\begin{array}{l}\text { Index is based on the } \\
\text { average duration and } \\
\text { frequency of recreational } \\
\text { activities and is then } \\
\text { expressed as metabolic } \\
\text { equivalent (MET) }\end{array}$ & $\begin{array}{l}\text { Lowest tertile } \\
\text { Middle tertile } \\
\text { Highest tertile }\end{array}$ & $\begin{array}{c}1.00 \\
1.28(0.65-2.51) \\
0.38(0.14-1.85)\end{array}$ & $\begin{array}{l}\text { Age, cigarette pack-years, } \\
\text { diabetes, systolic blood } \\
\text { pressure, HDL, triglycer- } \\
\text { ides, family history of } \\
\mathrm{CHD} \text {, socioeconomic } \\
\text { status, leucocytes, fibri- } \\
\text { nogen, haemoglobin, } \\
\text { apolipoprotein B and } \\
\text { ferritin }\end{array}$ & $5(2 ; 1 ; 2)$ \\
\hline $\begin{array}{l}\text { Eaton et al. (The Israeli } \\
\text { Ischemic Heart Disease } \\
\text { Study) [16] }\end{array}$ & Israel (1963) & $709 / 8463$ & 21 & $>40$ & M & $\begin{array}{l}\text { Death } \\
\text { from } \\
\text { CHD }\end{array}$ & $\begin{array}{l}\text { Physical activity is graded } \\
\text { into qualitative } \\
\text { categories }\end{array}$ & $\begin{array}{l}\text { Sedentary } \\
\text { Light } \\
\text { Light daily } \\
\text { Heavy }\end{array}$ & $\begin{array}{c}1.00 \\
0.79(0.63-0.99) \\
0.73(0.59-0.89) \\
0.71(0.52-0.98)\end{array}$ & Age & $2(0 ; 1 ; 1)$ \\
\hline $\begin{array}{l}\text { Folsom et al. (men) (The } \\
\text { Atherosclerosis Risk in } \\
\text { Communities Study) } \\
\text { [17] }\end{array}$ & USA (1987) & $223 / 6188$ & $4-7$ & $45-64$ & M & $\mathrm{CHD}$ & $\begin{array}{l}\text { Score is based on } \\
\text { the average duration, } \\
\text { frequency and type } \\
\text { of physical activity }\end{array}$ & $\begin{array}{l}\text { First quartile } \\
\text { Second quartile } \\
\text { Third quartile } \\
\text { Fourth quartile }\end{array}$ & $\begin{array}{c}1.00 \\
1.08(0.75-1.55) \\
0.83(0.51-1.36) \\
0.89(0.59-1.35)\end{array}$ & $\begin{array}{l}\text { Age, smoking habit, total } \\
\text { cholesterol, HDL, systo- } \\
\text { lic blood pressure, dia- } \\
\text { betes, waist-to-hip ratio, } \\
\text { education and alcohol }\end{array}$ & $6(2 ; 2 ; 2)$ \\
\hline $\begin{array}{l}\text { Folsom et al. (women) (The } \\
\text { Atherosclerosis Risk in } \\
\text { Communities Study) } \\
\text { [17] }\end{array}$ & USA (1987) & 97/7852 & $4-7$ & $45-64$ & $\mathrm{~F}$ & $\mathrm{CHD}$ & $\begin{array}{l}\text { Score is based on } \\
\text { the average duration, } \\
\text { frequency and type } \\
\text { of physical activity }\end{array}$ & $\begin{array}{l}\text { First quartile } \\
\text { Second quartile } \\
\text { Third quartile } \\
\text { Fourth quartile }\end{array}$ & $\begin{array}{c}1.00 \\
0.74(0.42-1.31) \\
1.07(0.55-2.09) \\
0.64(0.34-1.24)\end{array}$ & $\begin{array}{l}\text { Age, smoking habit, total } \\
\text { cholesterol, HDL, systo- } \\
\text { lic blood pressure, dia- } \\
\text { betes, waist-to-hip ratio, } \\
\text { education, alcohol and } \\
\text { hormone replacement } \\
\text { therapy }\end{array}$ & $6(2 ; 2 ; 2)$ \\
\hline $\begin{array}{l}\text { Rosengren and Wilhelm- } \\
\text { sen (The Multifactor Pri- } \\
\text { mary Prevention Study in } \\
\text { Goteborg) [18] }\end{array}$ & $\begin{array}{l}\text { Sweden } \\
(1970)\end{array}$ & $684 / 7142$ & 20 & $47-55$ & M & $\begin{array}{l}\text { Death } \\
\text { from } \\
\text { CHD }\end{array}$ & $\begin{array}{l}\text { Physical activity is graded } \\
\text { into qualitative } \\
\text { categories }\end{array}$ & $\begin{array}{l}\text { Sedentary } \\
\text { Moderately } \\
\text { active } \\
\text { Regular } \\
\text { exercise }\end{array}$ & $\begin{array}{c}1.00 \\
0.84(0.71-1.00) \\
0.84(0.73-0.96)\end{array}$ & $\begin{array}{l}\text { Age, body mass index, } \\
\text { smoking habit, diastolic } \\
\text { blood pressure, total } \\
\text { cholesterol, diabetes, } \\
\text { alcohol and occupation }\end{array}$ & $2(0 ; 1 ; 1)$ \\
\hline $\begin{array}{l}\text { Weller and Corey (Canada } \\
\text { Fitness Survey) [19] }\end{array}$ & $\begin{array}{l}\text { Canada } \\
(1981)\end{array}$ & $109 / 6620$ & 7 & $\begin{array}{c}>30 \text { (mean: } \\
50.3)\end{array}$ & $\mathrm{F}$ & Fatal MI & $\begin{array}{l}\text { Index of energy expenditure } \\
\text { is based on the duration } \\
\text { and frequency of recrea- } \\
\text { tional activities and is } \\
\text { then expressed in kcal } \times \\
\mathrm{kg} \times \text { day }\end{array}$ & $\begin{array}{l}\text { Sedentary } \\
\text { Moderately } \\
\text { active } \\
\text { Highly active }\end{array}$ & $\begin{array}{c}1.00 \\
0.78(0.45-1.34) \\
0.57(0.36-0.90)\end{array}$ & Age & $2(1 ; 1 ; 0)$ \\
\hline $\begin{array}{l}\text { Gartside et al. (NHANES I } \\
\text { Epidemiologic Follow-Up } \\
\text { Study) [13] }\end{array}$ & USA (1971) & $1958 / 5811$ & 16 & $25-74$ & $M / F$ & $\mathrm{CHD}$ & $\begin{array}{l}\text { Physical activity is graded } \\
\text { into qualitative cate- } \\
\text { gories }\end{array}$ & $\begin{array}{l}\text { None } \\
\text { Moderate } \\
\text { Intense }\end{array}$ & $\begin{array}{l}1.00 \\
0.81(P=0.002) \\
0.83(P=0.06)\end{array}$ & $\begin{array}{l}\text { Age, sex, smoking habit, } \\
\text { body mass index, } \\
\text { cholesterol, alcohol, } \\
\text { education and diet }\end{array}$ & $3(0 ; 1 ; 2)$ \\
\hline
\end{tabular}




\begin{tabular}{|c|c|c|c|c|c|c|c|c|c|c|c|}
\hline Source, year (cohort) & $\begin{array}{l}\text { Country } \\
\text { (baseline } \\
\text { year) }\end{array}$ & $\begin{array}{l}\text { Number of } \\
\text { events/ } \\
\text { number of } \\
\text { participants }\end{array}$ & $\begin{array}{l}\text { Follow-up, } \\
\text { years }\end{array}$ & Age, years & Sex & Outcome & $\begin{array}{l}\text { Assessment of physical } \\
\text { activity }\end{array}$ & $\begin{array}{l}\text { Physical activity } \\
\text { categories }\end{array}$ & $\begin{array}{l}\text { Adjusted RR } \\
(95 \% \mathrm{Cl})\end{array}$ & Adjustment & Study quality \\
\hline $\begin{array}{l}\text { Manson et al. (The Nurses' } \\
\text { Health Study) [20] }\end{array}$ & USA (1976) & $645 / 72488$ & 8 & $40-65$ & $F$ & $\mathrm{CHD}$ & $\begin{array}{l}\text { Index is based on the } \\
\text { average duration and } \\
\text { frequency of recreational } \\
\text { activities and is then } \\
\text { expressed as MET } \times \\
\text { hour } \times \text { week }\end{array}$ & $\begin{array}{l}\text { First quintile } \\
\text { Second quintile } \\
\text { Third quintile } \\
\text { Fourth quintile } \\
\text { Fifth quintile }\end{array}$ & $\begin{array}{l}1.00 \\
0.88(0.71-1.10) \\
0.81(0.64-1.02) \\
0.74(0.58-0.95) \\
0.66(0.51-0.86)\end{array}$ & $\begin{array}{l}\text { Age, body mass index, } \\
\text { smoking habit, hyperten- } \\
\text { sion, hypercholestero- } \\
\text { laemia, diabetes, } \\
\text { menopausal status, fa- } \\
\text { mily history of Ml, vitamin } \\
\text { use, alcohol and aspirin } \\
\text { use }\end{array}$ & $6(2 ; 2 ; 2)$ \\
\hline $\begin{array}{l}\text { Sesso et al. (The Harvard } \\
\text { Alumni Health Study) } \\
\text { [21] }\end{array}$ & USA (1977) & $\begin{array}{l}2135 / \\
12516\end{array}$ & 15 & $39-88$ & M & $\mathrm{CHD}$ & $\begin{array}{l}\text { Index of weekly energy } \\
\text { expenditure is derived } \\
\text { from several recreational } \\
\text { activities and is then } \\
\text { expressed in } \mathrm{kJ} / \text { week }\end{array}$ & $\begin{array}{l}\text { First quintile } \\
\text { Second quintile } \\
\text { Third quintile } \\
\text { Fourth quintile } \\
\text { Fifth quintile }\end{array}$ & $\begin{array}{c}1.00 \\
0.90(0.79-1.03) \\
0.81(0.71-0.92) \\
0.80(0.69-0.93) \\
0.81(0.71-0.94)\end{array}$ & $\begin{array}{l}\text { Age, body mass index, } \\
\text { smoking habit, hyper- } \\
\text { tension, diabetes, alcohol } \\
\text { and early parental death }\end{array}$ & $4(2 ; 0 ; 2)$ \\
\hline $\begin{array}{l}\text { Wannamethee et al. (The } \\
\text { British Regional Heart } \\
\text { Study) [22] }\end{array}$ & UK (1978) & $616 / 5159$ & 16.8 & $40-59$ & M & $\mathrm{CHD}$ & $\begin{array}{l}\text { Score is based on the } \\
\text { average duration, } \\
\text { frequency, and type } \\
\text { of physical activity, } \\
\text { according to the energy } \\
\text { demands }\end{array}$ & $\begin{array}{l}\text { Inactive } \\
\text { Occasional } \\
\text { Light } \\
\text { Moderate } \\
\text { Vigorous }\end{array}$ & $\begin{array}{l}1.00 \\
0.71(0.54-0.93) \\
0.79(0.61-1.05) \\
0.49(0.55-0.68) \\
0.89(0.66-1.18)\end{array}$ & $\begin{array}{l}\text { Age, smoking habit, body } \\
\text { mass index, diastolic } \\
\text { blood pressure, trigly- } \\
\text { cerides, HDL, insulin } \\
\text { and heart rate }\end{array}$ & $5(2 ; 1 ; 2)$ \\
\hline $\begin{array}{l}\text { Smith et al. (The Whitehall } \\
\text { Study) [9] }\end{array}$ & UK (1967) & $926 / 6702$ & 25 & $40-64$ & M & $\begin{array}{l}\text { Mortality } \\
\text { from } \\
\text { CHD }\end{array}$ & $\begin{array}{l}\text { Physical activity is graded } \\
\text { into qualitative categories }\end{array}$ & $\begin{array}{l}\text { Inactive } \\
\text { Moderately } \\
\text { active } \\
\text { Active }\end{array}$ & $\begin{array}{c}1.00 \\
1.03(0.83-1.25) \\
0.88(0.71-1.11)\end{array}$ & $\begin{array}{l}\text { Age, body mass index, } \\
\text { smoking habit, diastolic } \\
\text { blood pressure, diabetes } \\
\text { and glucose intolerance }\end{array}$ & $3(0 ; 1 ; 2)$ \\
\hline $\begin{array}{l}\text { Haapanen-Niemi et al. } \\
\text { (women) [10] }\end{array}$ & $\begin{array}{l}\text { Finland } \\
(1980)\end{array}$ & $37 / 1122$ & 16 & $35-63$ & $\mathrm{~F}$ & $\begin{array}{l}\text { Mortality } \\
\text { from } \\
\text { CHD }\end{array}$ & $\begin{array}{l}\text { Index of energy expenditure } \\
\text { is based on the duration } \\
\text { and frequency of recrea- } \\
\text { tional activities and } \\
\text { is then expressed as } \\
\text { kilocalories/week }\end{array}$ & $\begin{array}{l}\text { Low } \\
\text { Moderate } \\
\text { High }\end{array}$ & $\begin{array}{c}1.00 \\
2.32(0.86-6.25) \\
0.85(0.37-1.96)\end{array}$ & $\begin{array}{l}\text { Age, smoking habit, } \\
\text { socioeconomic status } \\
\text { and alcohol }\end{array}$ & $4(1 ; 1 ; 2)$ \\
\hline $\begin{array}{l}\text { Haapanen-Niemi et al. } \\
\text { (men) [10] }\end{array}$ & $\begin{array}{l}\text { Finland } \\
(1980)\end{array}$ & $78 / 1090$ & 16 & $35-63$ & M & $\begin{array}{l}\text { Mortality } \\
\text { from } \\
\text { CHD }\end{array}$ & $\begin{array}{l}\text { Index of energy expenditure } \\
\text { is based on the duration } \\
\text { and frequency of recrea- } \\
\text { tional activities and } \\
\text { is then expressed as } \\
\text { kilocalories/week }\end{array}$ & $\begin{array}{l}\text { Low } \\
\text { Moderate } \\
\text { High }\end{array}$ & $\begin{array}{c}1.00 \\
1.13(0.57-2.27) \\
0.59(0.31-1.11)\end{array}$ & $\begin{array}{l}\text { Age, smoking habit, socio- } \\
\text { economic status and } \\
\text { alcohol }\end{array}$ & $3(0 ; 1 ; 2)$ \\
\hline $\begin{array}{l}\text { Manson et al. (The Wo- } \\
\text { men's Health Initiative } \\
\text { Observational Study) [4] }\end{array}$ & USA (1994) & $345 / 73743$ & 5.9 & $50-79$ & $\mathrm{~F}$ & $\mathrm{CHD}$ & $\begin{array}{l}\text { Index is based on the } \\
\text { average duration and } \\
\text { frequency of recreational } \\
\text { activities and is then } \\
\text { expressed as MET } \times \\
\text { hour } \times \text { week }\end{array}$ & $\begin{array}{l}\text { First quintile } \\
\text { Second quintile } \\
\text { Third quintile } \\
\text { Fourth quintile } \\
\text { Fifth quintile }\end{array}$ & $\begin{array}{l}1.00 \\
0.73(0.53-0.99) \\
0.69(0.51-0.95) \\
0.68(0.50-0.93) \\
0.47(0.33-0.67)\end{array}$ & $\begin{array}{l}\text { Age, body mass index, } \\
\text { smoking habit, family } \\
\text { history of MI, meno- } \\
\text { pause, hormone, alcohol, } \\
\text { waist-to-hip ratio and } \\
\text { diet }\end{array}$ & $5(1 ; 2 ; 2)$ \\
\hline $\begin{array}{l}\text { Wagner et al. (The PRIME } \\
\text { Study) [23] }\end{array}$ & $\begin{array}{c}\text { France/UK } \\
(1991)\end{array}$ & $167 / 9758$ & 5 & $50-59$ & $\mathrm{M} / \mathrm{F}$ & $\mathrm{CHD}$ & $\begin{array}{l}\text { Index is based on the } \\
\text { average duration and } \\
\text { frequency of recreational } \\
\text { activities and is then ex- } \\
\text { pressed as MET } \times \\
\text { hour } \times \text { week }\end{array}$ & $\begin{array}{l}\text { Lowest tertile } \\
\text { Middle tertile } \\
\text { Highest tertile }\end{array}$ & $\begin{array}{c}1.00 \\
0.73(0.51-1.05) \\
0.66(0.46-0.96)\end{array}$ & $\begin{array}{l}\text { LTPA as a continuous } \\
\quad \text { variable }\end{array}$ & $4(2 ; 1 ; 1)$ \\
\hline $\begin{array}{l}\text { Tanasescu et al. (The } \\
\text { Health Professionals' } \\
\text { Study) [24] }\end{array}$ & USA (1986) & $\begin{array}{c}1700 / \\
44452\end{array}$ & 12 & $40-75$ & M & $\mathrm{CHD}$ & $\begin{array}{l}\text { Index is based on the } \\
\text { average duration and } \\
\text { frequency of recreational } \\
\text { activities and is then } \\
\text { expressed as MET } \times \\
\text { hour } \times \text { week }\end{array}$ & $\begin{array}{l}\text { Low } \\
\text { Moderate } \\
\text { High }\end{array}$ & $\begin{array}{c}1.00 \\
0.94(0.83-1.04) \\
0.83(0.74-0.97)\end{array}$ & $\begin{array}{l}\text { Age, body mass index, } \\
\text { smoking habit, hyper- } \\
\text { tension, hypercholester- } \\
\text { olaemia, diabetes, family } \\
\text { history of } \mathrm{Ml} \text {, alcohol and } \\
\text { nutrient intake }\end{array}$ & $5(1 ; 2 ; 2)$ \\
\hline
\end{tabular}




\begin{tabular}{|c|c|c|c|c|c|c|c|c|c|c|c|}
\hline $\begin{array}{l}\text { Yu et al. (The Caerphilly } \\
\text { study) [25] }\end{array}$ & UK (1984) & $82 / 1975$ & 10.5 & $49-64$ & $M$ & $\begin{array}{l}\text { Death } \\
\text { from } \\
\mathrm{CHD}\end{array}$ & $\begin{array}{l}\text { Activity index is derived } \\
\text { from a record of leisure } \\
\text { activities and is then } \\
\text { expressed as kilocal- } \\
\text { ories/day }\end{array}$ & $\begin{array}{l}\text { Light } \\
\text { Moderate } \\
\text { Heavy }\end{array}$ & $\begin{array}{c}1.00 \\
0.74(0.44-1.25) \\
0.55(0.31-0.98)\end{array}$ & $\begin{array}{l}\text { Age, body mass index, } \\
\text { smoking habit, diastolic } \\
\text { blood pressure, dia- } \\
\text { betes, family history of } \\
\text { CVD and social class }\end{array}$ & $5(2 ; 1 ; 2)$ \\
\hline $\begin{array}{l}\text { Sundquist et al. (Swedish } \\
\text { Annual Level-of-Living } \\
\text { Survey) [26] }\end{array}$ & $\begin{array}{l}\text { Sweden } \\
(1988)\end{array}$ & $373 / 5196$ & 11.7 & $35-74$ & $\mathrm{M} / \mathrm{F}$ & $\mathrm{CHD}$ & $\begin{array}{l}\text { Physical activity is graded } \\
\text { into qualitative cate- } \\
\text { gories }\end{array}$ & $\begin{array}{l}\text { First level } \\
\text { Second level } \\
\text { Third level } \\
\text { Fourth level }\end{array}$ & $\begin{array}{c}1.00 \\
0.76(0.55-1.07) \\
0.74(0.53-1.04) \\
0.59(0.37-0.95)\end{array}$ & $\begin{array}{l}\text { Age, sex, body mass index, } \\
\text { smoking habit and in- } \\
\text { come }\end{array}$ & $3(0 ; 1 ; 2)$ \\
\hline $\begin{array}{l}\text { Conroy et al. (The Wo- } \\
\text { men's Health Study) [27] }\end{array}$ & USA (1992) & $477 / 39876$ & 9 & $>45$ & $\mathrm{~F}$ & $\mathrm{CHD}$ & $\begin{array}{l}\text { Average time spent in } \\
\text { recreational activities } \\
\text { is derived and is then } \\
\text { expressed as } \mathrm{kcal} / \mathrm{week}\end{array}$ & $\begin{array}{l}\text { First quartile } \\
\text { Second quartile } \\
\text { Third quartile } \\
\text { Fourth quartile }\end{array}$ & $\begin{array}{c}1.00 \\
0.62(0.48-0.80) \\
0.61(0.48-0.79) \\
0.61(0.46-0.81)\end{array}$ & $\begin{array}{l}\text { Age, smoking habit, family } \\
\text { history of CVD, alcohol, } \\
\text { menopausal status, hor- } \\
\text { mone and diet }\end{array}$ & $6(2 ; 2 ; 2)$ \\
\hline $\begin{array}{l}\text { Noda et al. (The Japan } \\
\text { Collaborative Cohort } \\
\text { Study for Evaluation for } \\
\text { Cancer Risk) [11] }\end{array}$ & $\begin{array}{l}\text { Japan } \\
(1988)\end{array}$ & $397 / 73265$ & 9.7 & $40-79$ & $\mathrm{M} / \mathrm{F}$ & $\begin{array}{l}\text { Mortality } \\
\text { from } \\
\text { CHD }\end{array}$ & $\begin{array}{l}\text { Average daily time spent } \\
\text { in sports is derived and } \\
\text { is then grouped into } \\
\text { categories }\end{array}$ & $\begin{array}{l}\text { Lowest } \\
\text { category } \\
\text { Middle cate- } \\
\text { gory } \\
\text { Highest } \\
\text { category }\end{array}$ & $\begin{array}{c}1.00 \\
0.80(0.52-1.22) \\
0.51(0.32-0.82)\end{array}$ & $\begin{array}{l}\text { Age, sex, body mass index, } \\
\text { smoking habit, hyper- } \\
\text { tension, diabetes, alcohol, } \\
\text { education and fish }\end{array}$ & $4(1 ; 1 ; 2)$ \\
\hline $\begin{array}{l}\text { Schnohr et al. (The Co- } \\
\text { penhagen City Heart } \\
\text { Study) [28] }\end{array}$ & $\begin{array}{c}\text { Denmark } \\
(1976)\end{array}$ & $292 / 4894$ & 20 & $20-79$ & $\mathrm{M} / \mathrm{F}$ & $\mathrm{CHD}$ & $\begin{array}{l}\text { Physical activity is graded } \\
\text { into qualitative cate- } \\
\text { gories }\end{array}$ & $\begin{array}{l}\text { Low } \\
\text { Moderate } \\
\text { High }\end{array}$ & $\begin{array}{c}1.00 \\
0.71(0.51-0.99) \\
0.56(0.38-0.82)\end{array}$ & $\begin{array}{l}\text { Age, sex, body mass index, } \\
\text { smoking habit, hyper- } \\
\text { tension, total cholesterol, } \\
\text { HDL, diabetes, alcohol, } \\
\text { education and income }\end{array}$ & $3(0 ; 1 ; 2)$ \\
\hline $\begin{array}{l}\text { Vatten et al. (The HUNT } \\
\text { Study) (women) [12] }\end{array}$ & $\begin{array}{l}\text { Norway } \\
(1984)\end{array}$ & $\begin{array}{r}1202 / \\
34868\end{array}$ & 16 & $>20$ & $\mathrm{~F}$ & $\mathrm{CHD}$ & $\begin{array}{l}\text { Score is based on the } \\
\text { average duration, fre- } \\
\text { quency, and type of } \\
\text { physical activity, and } \\
\text { is then expressed as } \\
\text { qualitative categories }\end{array}$ & $\begin{array}{l}\text { Low } \\
\text { Moderate } \\
\text { High }\end{array}$ & $\begin{array}{c}1.00 \\
0.86(0.69-1.07) \\
0.69(0.54-0.88)\end{array}$ & $\begin{array}{l}\text { Age, body mass index, } \\
\text { smoking habit, hyper } \\
\text { tension, marital status, } \\
\text { education and alcohol }\end{array}$ & $4(1 ; 1 ; 2)$ \\
\hline $\begin{array}{l}\text { Vatten et al. (The HUNT } \\
\text { Study) (men) [12] }\end{array}$ & $\begin{array}{l}\text { Norway } \\
(1984)\end{array}$ & $\begin{array}{r}1923 / \\
32872\end{array}$ & 16 & $>20$ & M & $\mathrm{CHD}$ & $\begin{array}{l}\text { Score is based on the } \\
\text { average duration, fre- } \\
\text { quency, and type of } \\
\text { physical activity, and } \\
\text { is then expressed as } \\
\text { qualitative categories }\end{array}$ & $\begin{array}{l}\text { Low } \\
\text { Moderate } \\
\text { High }\end{array}$ & $\begin{array}{l}1.00 \\
0.95(0.77-1.18) \\
0.83(0.65-1.05)\end{array}$ & $\begin{array}{l}\text { Age, body mass index, } \\
\text { smoking habit, hyperten- } \\
\text { sion, marital status, edu- } \\
\text { cation and alcohol }\end{array}$ & $4(1 ; 1 ; 2)$ \\
\hline Hu et al. (Women) [29] & $\begin{array}{l}\text { Finland } \\
(1972)\end{array}$ & $\begin{array}{l}1587 / \\
23376\end{array}$ & 18.9 & $25-64$ & $\mathrm{~F}$ & $\mathrm{CHD}$ & $\begin{array}{l}\text { Physical activity is } \\
\text { graded into qualitative } \\
\text { categories }\end{array}$ & $\begin{array}{l}\text { Low } \\
\text { Moderate } \\
\text { High }\end{array}$ & $\begin{array}{c}1.00 \\
0.85(0.77-0.95) \\
0.77(0.62-0.96)\end{array}$ & $\begin{array}{l}\text { Age, body mass index, } \\
\text { smoking habit, systolic } \\
\text { blood pressure, total } \\
\text { cholesterol, diabetes, } \\
\text { alcohol, education and } \\
\text { other types of physical } \\
\text { activity }\end{array}$ & $4(1 ; 1 ; 2)$ \\
\hline Hu et al. (men) [29] & $\begin{array}{l}\text { Finland } \\
(1972)\end{array}$ & $\begin{array}{c}3073 / \\
19804\end{array}$ & 18.9 & $25-64$ & M & $\mathrm{CHD}$ & $\begin{array}{l}\text { Physical activity is } \\
\text { graded into qualitative } \\
\text { categories }\end{array}$ & $\begin{array}{l}\text { Low } \\
\text { Moderate } \\
\text { High }\end{array}$ & $\begin{array}{c}1.00 \\
0.95(0.88-1.02) \\
0.84(0.74-0.95)\end{array}$ & $\begin{array}{l}\text { Age, body mass index, } \\
\text { smoking habit, systolic } \\
\text { blood pressure, total } \\
\text { cholesterol, diabetes, } \\
\text { alcohol, education } \\
\text { and other types of } \\
\text { physical activity }\end{array}$ & $4(1 ; 1 ; 2)$ \\
\hline
\end{tabular}

$\mathrm{CHD}$, coronary heart disease; $\mathrm{Cl}$, confidence interval; $\mathrm{CVD}$, cardiovascular disease; HDL, high-density lipoprotein; LTPA, leisure-time physical activity; MI, myocardial infarction; RR, relative risk. 
population. The moderate category of LTPA was determined by considering the middle category from the studies. The low level of LTPA consisted of the lowest category of LTPA among all the studies included, and it represented the reference category. We used the results of the original studies that involved multivariable models with the most complete adjustment for potential confounders; the confounding variables included in this analysis are shown in Table 1 . For studies using high LTPA as the referent [9-12], we recalculated the relative risk, with low LTPA as the reference category, using conventional procedures. For the study by Gartside $e t$ al. [13], only a relative risk and a $P$ value were provided. The $P$ value was, thus, first converted into corresponding twosided $:$ statistics (from a table of standard normal variates), from which unreported standard errors were calculated. We used a random-effects model, which accounts for the interstudy variation and provides a more conservative effect than the fixed model. Estimates of the random-summary risk ratios, with $95 \%$ CIs, from all the studies were obtained by combining the separate estimates of the inverse-variance-weighted log risk ratios from each study.

The $Q$ statistic, a test of homogeneity between studies, was calculated. In addition, the statistic $I^{2}$ was used to investigate heterogeneity by examining the extent of inconsistency across the study results. To examine the potential source of heterogeneity across studies for the high level of physical activity, we conducted sensitivity analyses according to some of the characteristics of the studies included in the meta-analysis. These characteristics were sex (men/women), different regions of origin of the studies (USA/Europe), study quality (scores above and below the mean score obtained by all the studies, that is, 5 points), duration of follow-up (above and below the mean duration of 13 years) and the method used for physical activity assessment (quantitative method: evaluation of intensity, duration and frequency of physical activity, expressed as an index of metabolic expenditure or qualitative method: nonstandardized categories of intensity of physical activity).

Funnel plots were generated, to assess potential publication biases. Moreover, to estimate whether publication biases (if present) could explain the observed associations, we calculated fail-safe numbers. These numbers indicate the number of studies with null results that would need to be added to the meta-analysis, to reduce the overall significant observed result to nonsignificance.

\section{Results}

Our search strategy generated 163 potentially relevant articles (Fig. 1). Eighty-eight studies were excluded because they evaluated only occupational physical activity $(n=17)$, because of study design $(n=44)$ or
Fig. 1
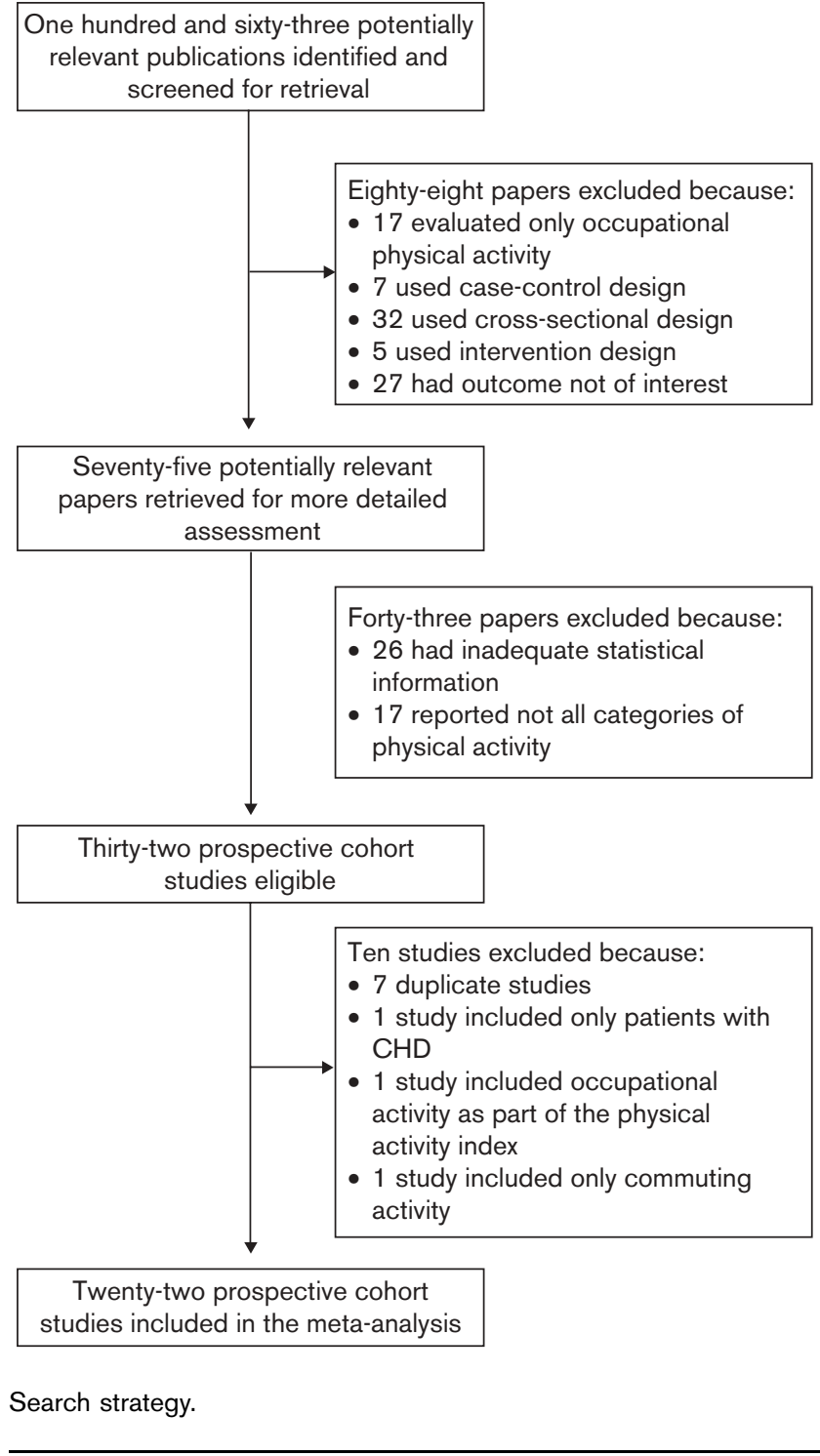

because they did not report the outcome of interest for this meta-analysis $(n=27)$. The foci of the latter studies might have been CVD as a whole, stroke or cerebrovascular accidents, or intermediate end points such as intima media thickness. Afterwards, a further 43 studies were eliminated because either the statistical information was not sufficient to compute an estimate of risk or its standard error for all the categories of physical activity $(n=26)$ or the study considered only one category of physical activity $(n=17)$. Finally, one study was eliminated because it also included patients with a preexisting coronary artery disease. Seven studies contained duplicate data from the same cohort study, so only complete or updated papers were included. One study was excluded because it reported only information on commuting physical activity, and another because the index of 
physical activity included occupational activity. As a result, 26 studies consisting prospective studies of 22 cohorts met the inclusion criteria (four studies had separate cohorts for men and women, which were entered as two independent groups). These studies enabled the incorporation of 513472 individuals, followed up for periods ranging from 4 to 25 years, reporting 20666 incident cases of CHD [4,9-29].

Characteristics of the studies included in the metaanalysis are described in Table 1. Interestingly, the studies included were more prevalently conducted either in North America (USA [4,13,14,17,20,21,24,27] and Canada [19]), or in North Europe (UK [9,22,23,25] and the Scandinavian countries $[10,12,15,18,26,29])$. With regard to assessment of physical activity, 14 studies recorded LTPA only through a qualitative questionnaire.
In other words, the quantum of physical activity was either grouped into nonstandardized categories or a score computed from the authors [9,11-13,16-18,22,26,28,29] was used. Furthermore, apart from three papers $[16,19,23]$, all the studies reported estimates of association after adjusting for most of the traditional and emerging cardiovascular risk factors.

Figure 2 shows the analysis of high-category LTPA compared with the sedentary category. The summary RRs in a random-effects model showed that individuals who reported performing a high level of physical activity had a significantly reduced risk of CHD (RR, 0.73; 95\% CI, 0.66-0.80; $P<0.00001)$. The $P$ value for heterogeneity, however, was also highly significant $(P<0.0001$; $I^{2}=73.2 \%$ ). The study by Wannamethee et al. [22], which accounted for the highest estimate of protection

Fig. 2

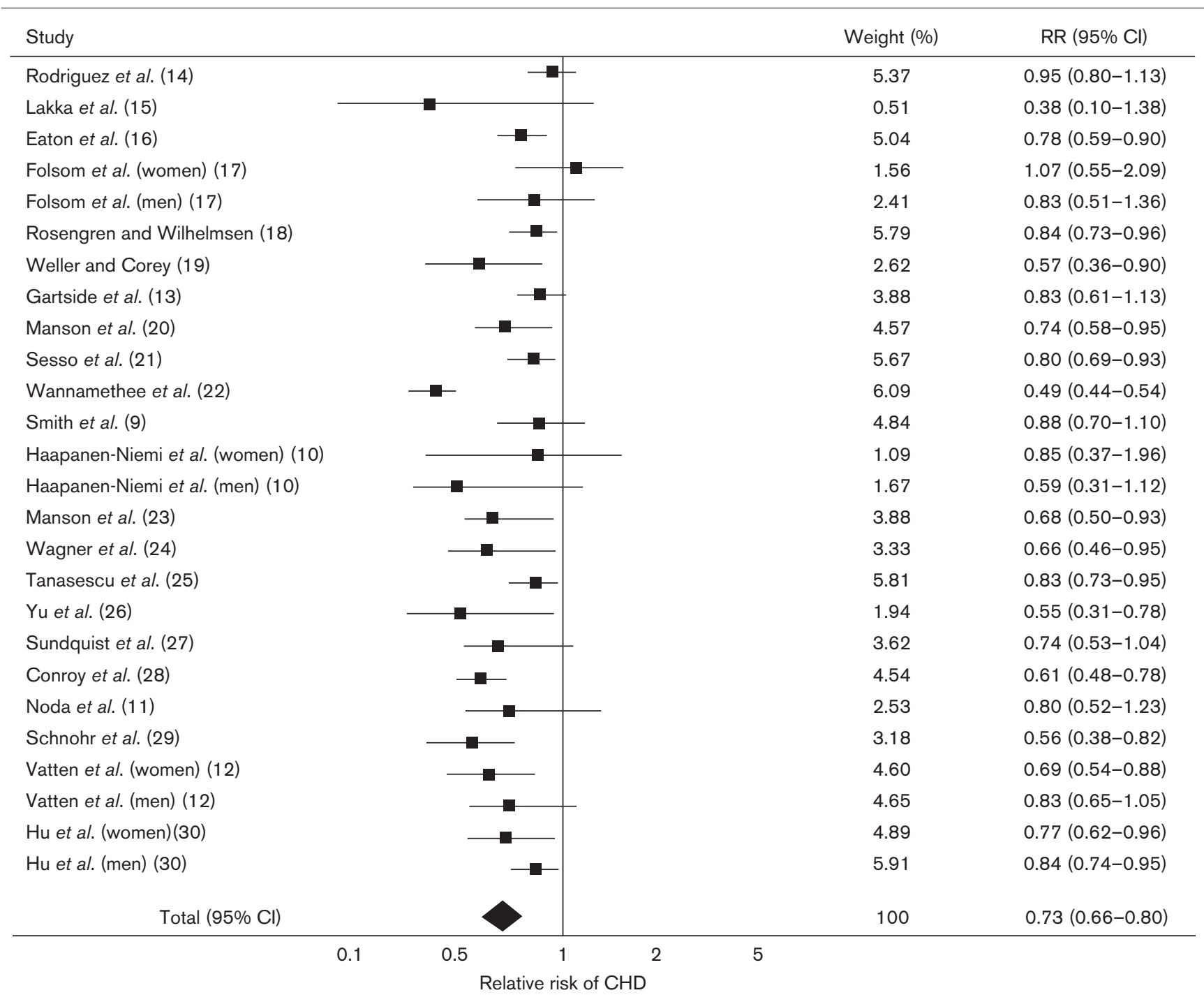

Risk of coronary heart disease for high, compared with low or nil, physical activity during leisure time. 
versus CHD, contributed substantially to the heterogeneity in this result. The exclusion of this study from the analysis did not, however, change the significant protection found between the high level of physical activity and CHD (RR, 0.78; 95\% CI, 0.74-0.82; $P<0.0001)\left(P\right.$ for heterogeneity $\left.=0.2 ; I^{2}=15.8 \%\right)$.

Subsequently, we compared the moderate level of physical activity versus the reference category: the overall effect of protection for a moderate amount of LTPA versus CHD remained statistically significant (RR, 0.88; 95\% CI, $0.83-0.93 ; P<0.00001)$ ( $P$ for heterogeneity: $0.05 ; I^{2}=33.4 \%$ ) (Fig. 3 ). A significant heterogeneity across the studies was present, mainly due to the study by Wannamethee et al. [22]. Even after the exclusion of this study, however, the significant protection of a moderate amount of LTPA versus CHD did not change substan- tially (RR, $0.89 ; 95 \%$ CI, $0.84-0.94 ; P<0.0001$ ) ( $P$ for heterogeneity $=0.07 ; I^{2}=30.7 \%$ ).

Furthermore, we conducted separate analyses, by taking into consideration studies with similar characteristics, such as sex, country of cohort, study quality, duration of follow-up and methods used for the assessment of physical activity (Table 2). In all, the different subanalyses of both the high and the moderate categories of intensity of LTPA were significantly associated with a reduced risk of CHD, with no significant influence of either variable analysed.

Finally, we explored the potential for publication bias using funnel plots of effect size versus standard error. The plots for both the categories of physical activity were broadly symmetrical, consistent with the conclusion that

Fig. 3

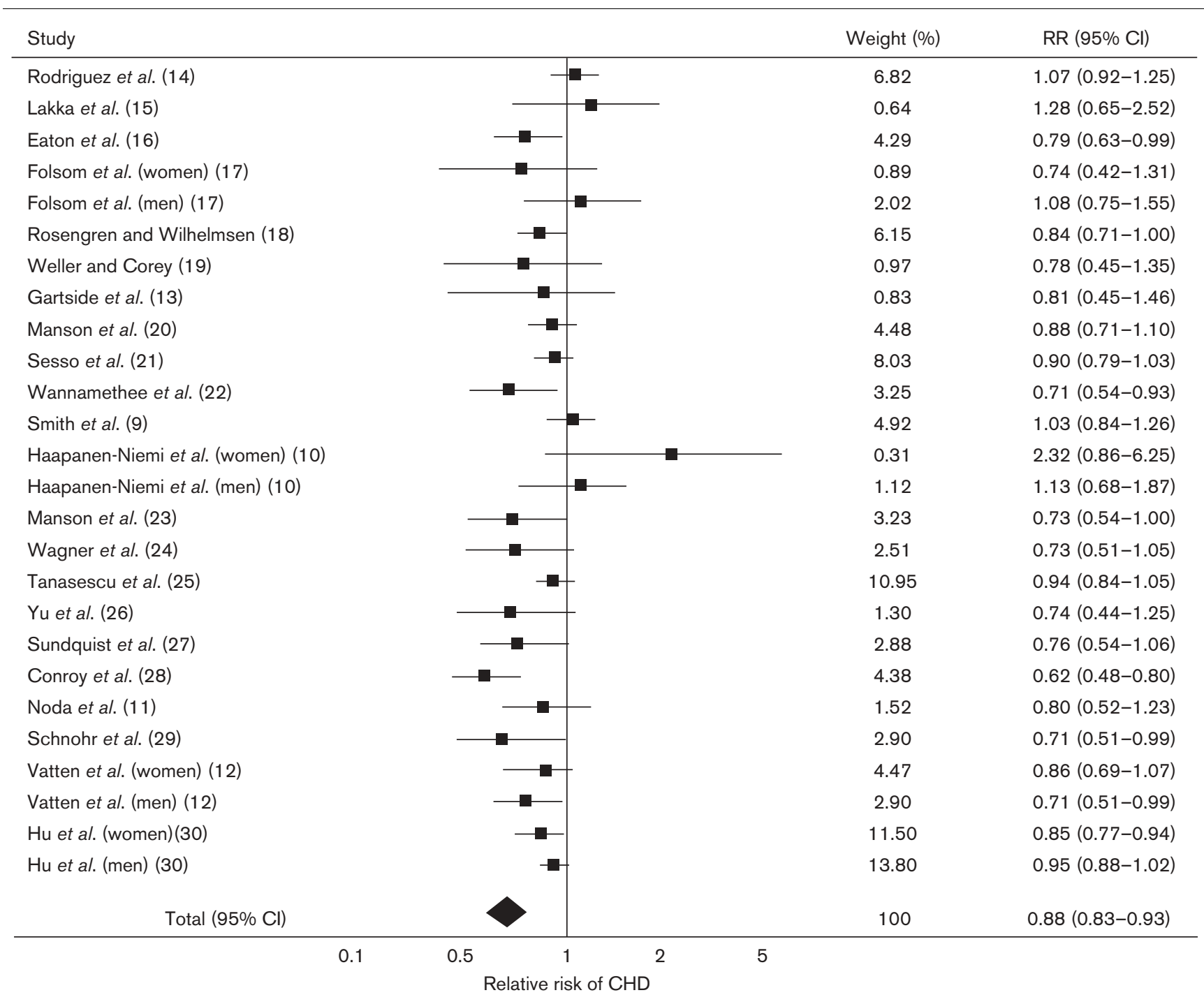

Risk of coronary heart disease for moderate, compared with low or nil, physical activity during leisure time. 
Table 2 Subgroup analysis

\begin{tabular}{|c|c|c|c|}
\hline & $\begin{array}{l}\text { No. of } \\
\text { studies }\end{array}$ & $\begin{array}{l}\text { High category of } \\
\text { physical activity }\end{array}$ & $\begin{array}{c}\text { Moderate category of } \\
\text { physical activity }\end{array}$ \\
\hline \multicolumn{4}{|l|}{ Sex } \\
\hline Men & 13 & $0.76(0.66-0.88)$ & $0.93(0.88-0.99)$ \\
\hline Women & 8 & $0.77(0.69-0.85)$ & $0.90(0.83-0.98)$ \\
\hline \multicolumn{4}{|l|}{ Country of cohort } \\
\hline USA & 9 & $0.80(0.73-0.87)$ & $0.88(0.79-0.98)$ \\
\hline Europe & 14 & $0.71(0.60-0.82)$ & $0.88(0.82-0.95)$ \\
\hline \multicolumn{4}{|l|}{ Study quality } \\
\hline$\geq 5$ points & 10 & $0.71(0.57-0.87)$ & $0.86(0.75-0.98)$ \\
\hline$<5$ points & 16 & $0.79(0.74-0.83)$ & $0.89(0.85-0.94)$ \\
\hline \multicolumn{4}{|l|}{ Duration of follow-up } \\
\hline $\begin{array}{l}\text { Follow-up of } 13 \\
\text { years or more }\end{array}$ & 14 & $0.75(0.66-0.86)$ & $0.90(0.85-0.96)$ \\
\hline $\begin{array}{c}\text { Follow-up of less } \\
\text { than } 13 \text { years }\end{array}$ & 12 & $0.71(0.64-0.79)$ & $0.83(0.74-0.92)$ \\
\hline \multicolumn{4}{|l|}{$\begin{array}{l}\text { Method of assess- } \\
\text { ment of physical } \\
\text { activity }\end{array}$} \\
\hline $\begin{array}{l}\text { Quantitative } \\
\text { assessment }\end{array}$ & 12 & $0.75(0.68-0.83)$ & $0.88(0.79-0.99)$ \\
\hline $\begin{array}{l}\text { Qualitative } \\
\text { assessment }\end{array}$ & 14 & $0.74(0.64-0.85)$ & $0.88(0.83-0.93)$ \\
\hline
\end{tabular}

there were no major publication biases. Moreover, we calculated the number of studies with null results that would need to be added to the meta-analysis to reduce the overall observed associations to nonsignificance, that is, the fail-safe number. This number was 876 for the high level of physical activity and 119 for the moderate level. Therefore, the number was robust for both categories, according to the commonly used criterion that an analysis requires a fail-safe number greater than $5 n+10$ (where $n$ is the original number of studies in the analysis).

\section{Discussion}

The current meta-analysis has quantitatively assessed the relationship between LTPA and CHD risk in a summary analysis of prospective studies of 22 cohorts, which included an overall population of more than 510000 healthy individuals, followed up for periods ranging from 4 to 25 years and reporting more than 20000 incident cases. The main finding of this study is that a moderateto-high level of LTPA is associated with a reduced risk of CHD. Indeed, compared with individuals performing low levels of or nil LTPA, highly and moderately active individuals had, respectively, a 27 and $12 \%$ lower risk of CHD incidence or mortality. This dose-dependent protective role of physical activity was found to be independent of several possible confounding variables, such as sex, methods used to assess physical activity and study quality.

During the last few years, there has been increasing interest in the identification of the possible risk factors contributing to the pathogenesis of CHD, and in the search for effective measures to reduce the risk burden for the whole population. Among all these intervention methods, physical activity has gained a relevant role owing to its efficacy and low cost. Indeed, many prospective and case-control studies have demonstrated that physical activity has a clear beneficial effect on the occurrence and progression of CVD.

The mechanisms by which regular physical activity confers such significant protection are different; in particular, regular exercise helps in controlling many established atherosclerotic risk factors, including elevated blood pressure, insulin resistance and glucose intolerance, elevated triglyceride concentrations, low high-density lipoprotein cholesterol concentrations and obesity. Furthermore, evidence indicates that increasing levels of physical activity are associated with lower circulating concentrations of inflammatory and haemostatic markers, and that exercise induces an enhancement of endothelial progenitor cells, with a significant improvement in the endothelial function. With regard to blood pressure, a meta-analysis [30] has recently demonstrated that aerobic exercise has a substantial blood pressure-lowering effect. In contrast, regarding the beneficial effect on lipid profile, data from a meta-analysis by Kelley et al. [31] reported that physical exercise increases high-density lipoprotein and reduces triglycerides in adults aged 50 years and older.

Over the last few decades, several reviews and metaanalyses have examined the relationship between physical activity and CHD. The scope of all these meta-analyses was not, however, to evaluate the role of LTPA in the primary prevention of CHD. In fact, they either evaluated CVD as a whole outcome, including both stroke and peripheral arterial disease, or included studies with data limited to some specific characteristics, such as men only or women only. Consequently, as of now, this metaanalysis is the most up-to-date, comprehensive assessment of the relationship between recreational physical activity and CHD among follow-up cohort studies. The meta-analytic technique used for evaluating the longterm effects of a specific variable among cohorts in prospective studies is a potentially powerful approach and should eliminate the selection and recall biases present in case-control studies. In this meta-analysis, in fact, we included cohort studies with large sample sizes and long durations of follow-up, by evaluating rigorously the methodology of the studies. As a result, many studies that were entered into previous meta-analyses were not used in our analysis. This choice helped us to obtain results with high confidence levels from the most reliable studies. Furthermore, the study quality was checked for all the studies included in the final analysis, with the use of a validated quality questionnaire, and the possible presence of publication bias was detected using two different methods, namely, funnel plot and fail-safe number. 
Our meta-analysis has some potential limitations. First, as in all observational studies of physical activity and the disease, exclusion of potential biases due to other components of lifestyle, such as diet, is extremely difficult. The beneficial effect of a healthy diet on the occurrence of CHD has been largely demonstrated [32] and, commonly, individuals who regularly practise physical activity also eat in a healthier way, thereby causing a bias in the results. Most of the studies included in our meta-analysis, however, have been adjusted for major confounding factors, including dietary factors in some, and the potential bias due to dietary habits could be somewhat reduced. In contrast, meta-analysis cannot solve the problems with confounding factors that might be present in the selected studies. Second, the method of assessment of physical activity varied substantially within the studies included in the final analysis. In particular, half of the studies estimated the amount of physical activity through a qualitative or semiqualitative questionnaire. Up to now, the most reliable and valid method used for the assessment of LTPA seems to be the quantitative measurements that report the intensity of physical activity $[33,34]$. Not all the studies available in the literature, however, used this method. This could lead to a potential overestimation of the results. In contrast, the subanalysis performed according to the methods used for the assessment of physical activity demonstrated that the significant protective effect of physical activity was not influenced by the method used for questionnaires.

Third, we decided to group the different levels of physical activity into three different categories: we took into consideration, for the high category of intensity, the second-highest levels, not the maximal, of LTPA from the selected studies. Otherwise the high category of intensity would have been achieved only by a limited proportion of the study population. This could have imparted a significant bias to the results, mainly arising from the elimination of the vigorous category. Rather, the effect of vigorous LTPA on the occurrence of CVD is debated: some studies have evidenced a clear beneficial role against the occurrence of cardiac events [4,20,21]; whereas, other studies have demonstrated that prolonged strenuous exercise induces an activation of the sympathetic system, of the blood-coagulation cascade as well as of the inflammatory pathway, thereby posing an increased risk for intravascular thrombosis and acute ischaemic events [35]. Moreover, in the 'real world', it is important to clearly assess the protective effect of a medium level of physical activity, a level that can be attained on a large scale by ordinary people, and one usually recommended by guidelines.

In conclusion, this meta-analysis provides convincing evidence on the significant protective role of moderate physical activity against CHD and gives strong specific support to the relevance of and urgency for action, which is possible by involving governments in widely implementing health-educational profiles, including the induction of moderate levels of LTPA. If these goals were achieved, CHD morbidity and mortality would be greatly reduced, with a significant reduction in national health expenditure.

\section{Acknowledgements}

Author contributions were as follows: Dr Sofi had full access to all of the data in the study and takes responsibility for the integrity of the data and the accuracy of the data analysis. The study concept and design were executed by Sofi, Francesco; Capalbo, Andrea and Gensini Gian Franco. Acquisition of data was carried out by Sofi, Francesco; Capalbo, Andrea and Cesari, Francesca. The analysis and the interpretation of the data were carried out by Sofi, Francesco. The drafting of the manuscript was by Sofi, Francesco; Capalbo, Andrea; Abbate, Rosanna and Cesari, Francesca. A critical revision of the manuscript for important intellectual content was carried out by Gensini, Gian Franco and Abbate, Rosanna. Study supervision was by Abbate, Rosanna. Financial disclosures: none declared.

\section{References}

1 World Health Organization. The World Health Report 2006: working together for health. http://www.who.int/whr/2006/whr06_en.pdf (May 8, 2007).

2 Sesso HD, Paffenbarger RS, Ha T, Lee IM. Physical activity and cardiovascular disease risk in middle-aged and older women. Am J Epidemiol 1999; 150:408-416.

3 Kannel WB, Belanger A, D'Agostino R, Israel I. Physical activity and physical demand on the job and risk of cardiovascular disease and death: the Framingham Study. Am Heart J 1986; 112:820-826.

4 Manson JA, Greenland P, LaCroix A, Stefanick ML, Mouton CP, Oberman A et al. Walking compared with vigorous exercise for the prevention of cardiovascular events in women. N Engl J Med 2002; 347:716-725.

5 Berlin JA, Colditz GA. A meta-analysis of physical activity in the prevention of coronary heart disease. Am J Epidemiol 1990; 132:612-628.

6 Powell KE, Thompson PD, Caspersen CJ, Kendrick JS. Physical activity and incidence of coronary heart disease. Ann Rev Public Health 1987; 8:253-287.

7 Eaton CB. Relation of physical activity and cardiovascular fitness to coronary heart disease, part I: a meta-analysis of the independent relation of physical activity and coronary heart disease. J Am Board Fam Pract 1992 5:31-42.

8 Oguma Y, Shinoda-Tagawa T. Physical activity decreases cardiovascular disease risk in women. Review and meta-analysis. Am J Prev Med 2004 26:407-418.

9 Smith DG, Shipley MJ, Batty GD, Morris JN, Marmot M. Physical activity and cause-specific mortality in the Whitehall study. Pub Health 2000; 114:308-315.

10 Haapanen-Niemi N, Miilunpalo S, Pasanen M, Vuori I, Oja P, Malmberg J. Body mass index, physical inactivity and low level of physical fitness as determinants of all-cause and cardiovascular disease mortality: $16 \mathrm{y}$ follow up of middle-aged and elderly men and women. Int J Obes Relat Metab Disord 2000; 24:1465-1474.

11 Noda $\mathrm{H}$, Iso $\mathrm{H}$, Toyoshima H, Date C, Yamamoto A, Kikuchi S, et al.; the JACC study group. Walking and sports participation and mortality from coronary heart disease and stroke. J Am Coll Cardiol 2005; 46:1761-1767.

12 Vatten LJ, Nilsen TIL, Romundstad PR, Droyvold WB, Holmen J. Adiposity and physical activity as predictors of cardiovascular mortality. Eur J Cardiovasc Prev Rehabil 2006; 13:909-915. 
13 Gartside PS, Wang P, Glueck CJ. Prospective assessment of coronary heart disease risk factors: the NHANES I epidemiologic follow-up study (NHEFS) 16-year follow-up. J Am Coll Nutr 1998; 17:263-269.

14 Rodriguez BL, Curb JD, Burchfiel CM, Abbott RD, Petrovitch H, Masaki K, et al. Physical activity and 23-year incidence of coronary heart disease morbidity and mortality among middle-aged men. Circulation 1994; 89:2540-2544

15 Lakka TA, Venalainen JM, Rauramaa R, Salonen R, Tuomilehto J, Salonen JT. Relation of leisure-time physical activity and cardiorespiratory fitness to the risk of acute myocardial infarction in men. N Engl J Med 1994; 330:1549-1554.

16 Eaton CB, Medalie JH, Flocke SA, Zyzanski SJ, Yaari S, Goldbourt U. Self-reported physical activity predicts long-term coronary heart disease and all-cause mortality. Twenty-one-year follow-up of the Israeli Ischemic Heart Disease Study. Arch Fam Med 1995; 4:323-329.

17 Folsom AR, Arnett DK, Hutchinson RG, Liao F, Clegg LX, Cooper LS. Physical activity and incidence of coronary heart disease in middle-aged women and men. Med Sci Sports Exerc 1997; 29:901-909.

18 Rosengren A, Wilhelmsen L. Physical activity protects against coronary death and deaths from all causes in middle-aged men. Evidence from a 20-year follow-up of the primary prevention study in Goteborg. Ann Epidemiol 1997; 7:69-75.

19 Weller I, Corey P. The impact of excluding non-leisure energy expenditure on the relation between physical activity and mortality in women. Epidemiology 1998; 9:632-635.

20 Manson JA, Hu FB, Rich-Edwards JW, Colditz GA, Stampfer MJ, Willett WC et al. A prospective study of walking as compared with vigorous exercise in the prevention of coronary heart disease in women. N Engl J Med 1999; 341:650-658.

21 Sesso HD, Paffenbarger RS, Lee IM. Physical activity and coronary heart disease in men. The Harvard Alumni Health Study. Circulation 2000; 102:975-980.

22 Wannamethee SG, Shaper G, Alberti KGMM. Physical activity, metabolic factors, and the incidence of coronary heart disease and type 2 diabetes. Arch Intern Med 2000; 160:2108-2116.

23 Wagner A, Simon C, Evans A, Ferrieres J, Montaye M, Ducimetiere P, Arveiler D; on behalf of the PRIME study group. Physical activity and coronary heart event incidence in Northern Ireland and France. Circulation 2002; 105:2247-2252.
24 Tanasescu M, Leitzmann MF, Rimm EB, Willett WC, Stampfer MJ, Hu FB. Exercise type and intensity in relation to coronary heart disease in men. JAMA 2002; 288:1994-2000.

25 Yu S, Yarnell YWG, Sweetnam PM, Murray L. What level of physical activity protects against premature cardiovascular death? The Caerphilly study. Heart 2003; 89:502-506.

26 Sundquist K, Qvist J, Johansson SE, Sundquist J. The long-term effect of physical activity on incidence of coronary heart disease: a 12-year follow-up study. Prev Med 2005; 41:219-225.

27 Conroy MB, Cook NR, Manson JE, Buring JE, Lee IM. Past physical activity, current physical activity, and risk of coronary heart disease. Med Sci Sports Exerc 2005; 37:1251-1256.

28 Schnohr $\mathrm{P}$, Lange $\mathrm{P}$, Scharling $\mathrm{H}$, Jensen JS. Long-term physical activity in leisure time and mortality from coronary heart disease, stroke, respiratory diseases, and cancer. The Copenhagen City Heart Study. Eur J Cardiovasc Prev Rehabil 2006; 13:173-179.

29 Hu G, Jousilahti P, Borodulin K, Barengo NC, Lakka TA, Nissinen A, et al. Occupational, commuting and leisure-time physical activity in relation to coronary heart disease among middle-aged Finnish men and women. Atherosclerosis 2006; [Epub Ahead of Print].

30 Fagard RH, Cornelissen VA. Effect of exercise on blood pressure control in hypertensive patients. Eur J Cardiovasc Prev Rehabil 2007; 14:12-17.

31 Kelley GA, Kelley KS, Tran ZV. Exercise, lipids, and lipoproteins in older adults: a meta-analysis. Prev Cardiol 2005; 8:206-214.

32 Trichopoulou A, Bamia C, Trichopoulos D. Mediterranean diet and survival among patients with coronary heart disease in Greece. Arch Intern Med 2005; 165:929-935.

33 Thompson PD, Buchner D, Pina IL, Balady GJ, Williams MA, Marcus BH, et al. Exercise and physical activity in the prevention and treatment of atherosclerotic cardiovascular disease. A statement from the council on clinical cardiology (subcommittee on exercise, rehabilitation, and prevention) and the council on nutrition, physical activity, and metabolism (subcommittee on physical activity). Circulation 2003; 107:3109-3116.

34 Giannuzzi P, Mezzani A, Saner H, Bjornstad H, Fioretti P, Mendes M, et al. Physical activity for primary and secondary prevention. Position paper of the working group on cardiac rehabilitation and exercise physiology of the European Society of Cardiology. Eur J Cardiovasc Prev Rehab 2003; 10:319-327.

35 Koenig W, Ernst E. Exercise and thrombosis. Coron Artery Dis 2000; 11:123-127. 